\title{
PlantGM: a database for genetic markers in rice (Oryza sativa) and Chinese cabbage (Brassica rapa)
}

\author{
Chang Kug Kim¹, Jung Sun Kim², Gang Seob Lee ${ }^{3}$, Beom Seok Park² and Jang Ho Hahn", * \\ ${ }^{1}$ Bioinformatics Division, National Institute of Agricultural Biotechnology (NIAB), Suwon 441-707, Korea; ${ }^{2}$ Brassica Genomics Team, \\ NIAB, Korea; ${ }^{3}$ Cell and Genetics Division, NIAB, Korea; JangHo Hahn* - E-mail: jhhahn@rda.go.kr; ${ }^{*}$ Corresponding author
}

received September 04, 2008; accepted September 21, 2008; published October 17, 2008

\begin{abstract}
:
The plant genetic map database (PlantGM) has been developed as a web-based system for providing information about genetic markers in rice (Oryza sativa) and Chinese cabbage (Brassica rapa). The database has three major parts and functions; (1) map search, (2) marker search, and (3) QTL Search. The database provides characterization information for about 3,258 genetic markers. It has 2,800 RFLP and 112 QTL markers related for rice in addition to 321 RFLP and 25 PCRbased markers for Chinese cabbage. In addition, a genetic linkage map was also constructed by using 1,054 markers from 2,912 markers in rice.
\end{abstract}

Availability: The database is available for free at http://www.niab.go.kr/nabic/PlantGM

Keywords: genetic map; genetic marker; QTL marker; rice; Chinese cabbage

\section{Background:}

Rice (Oryza sativa) and Chinese cabbage (Brassica rapa) are two of the most important crops in Korea and northeast Asia. Several databases have been constructed: rice [1], Chinese cabbage [2], and rice QTL markers [3] to increase the importance of genetic marker. In rice, several linkage maps based on various genetic markers have been constructed for the purpose of displaying positional relationships among mapped markers in each chromosome [4], identifying a candidate gene by QTL information [5], and matching a gene by combining mapping information [6]. In Chinese cabbage, the maps have been constructed in order to display the 139 RFLP loci organized into ten linkage groups [7] and, to contain the 545 sequence-tagged loci covering $1287 \mathrm{cM}$ [8]. The National Institute of Agricultural Biotechnology (NIAB) has developed a webbased database system to provide information about genetic markers in rice and Chinese cabbage. A subscriber is assisted in tracing new structures of the chromosome and gene positional functions through comparisons using specific genetic markers.

\section{Methodology:}

\section{Dataset}

The information on markers was collected from Brassica rapa genome project [2], rice genome project (Oryza sativa, Tongil: Indica/Japonica) of NIAB [9] in Korea. The markers related dataset consist 3,258 markers for rice and Chinese cabbage. It has 2,800 RFLP and 112 QTL markers related to rice an addition of 321 RFLP and 25 PCR-based markers related to Chinese cabbage (Brassica rapa). Additional information on genetic markers was collected from GRAMENE database for comparison [10].

\section{Development}

The platform was developed using MYSQL in JAVA. Data was stored in an Oracle relational database management system (RDBMS). We have developed a genetic linkage map at two levels, namely, (i) polymorphic markers (ii) QTL markers. We have analyzed data from 942 polymorphic markers by Kosambi function to construct a genetic map for polymorphic markers [11]. The map has been developed by INE (INtegrated rice genome Explorer) which displays positional relationships among mapped markers in each chromosome [4]. We also have analyzed data from 112 QTL markers by Haldane mapping function to construct a genetic map for QTL markers [12]. The final integrated map was constructed by using 1,054 markers in each of the 12 chromosomes among 2,912 markers of rice.

\section{Implementation and features:}

The PlantGM database is a web-based system that will provide information about genetic markers in rice and Chinese cabbage. The database has three major parts and functions namely, map search, marker search and QTL search. The individual data tables are linked to each other through their respective hypertext as shown in Figure 1. A user can access the individual characterization table by clicking a specific marker name on the linked hypertext in search result table (Figure 1). The 'name' field provides a detailed table (Figure 1a), which has a records about organism name, genus, species, subspecies, description, reference and image. The double click based hypertext 


\section{Bioinformation}

views the characterization information with RFLP-PCR image of Chinese cabbage where applicable (Figure 1b).

Discussion:

The PlantGM database provides the marker characterization information with phenotype image for rice and Chinese cabbage. The database is constantly updated and hence gives an up-to-date collection. There are 3,258 specific markers which are readily available in the database for rice and Chinese cabbage. A genetic linkage map database system in rice was constructed using the collected 1,054 markers. The genetic map provides locus of marker with linked information for polymorphic markers and QTL marker. It is possible to detect specific markers on each of the 12 chromosomes and the information present with associated trait and a mapped locus on a genetic map in rice using the system.

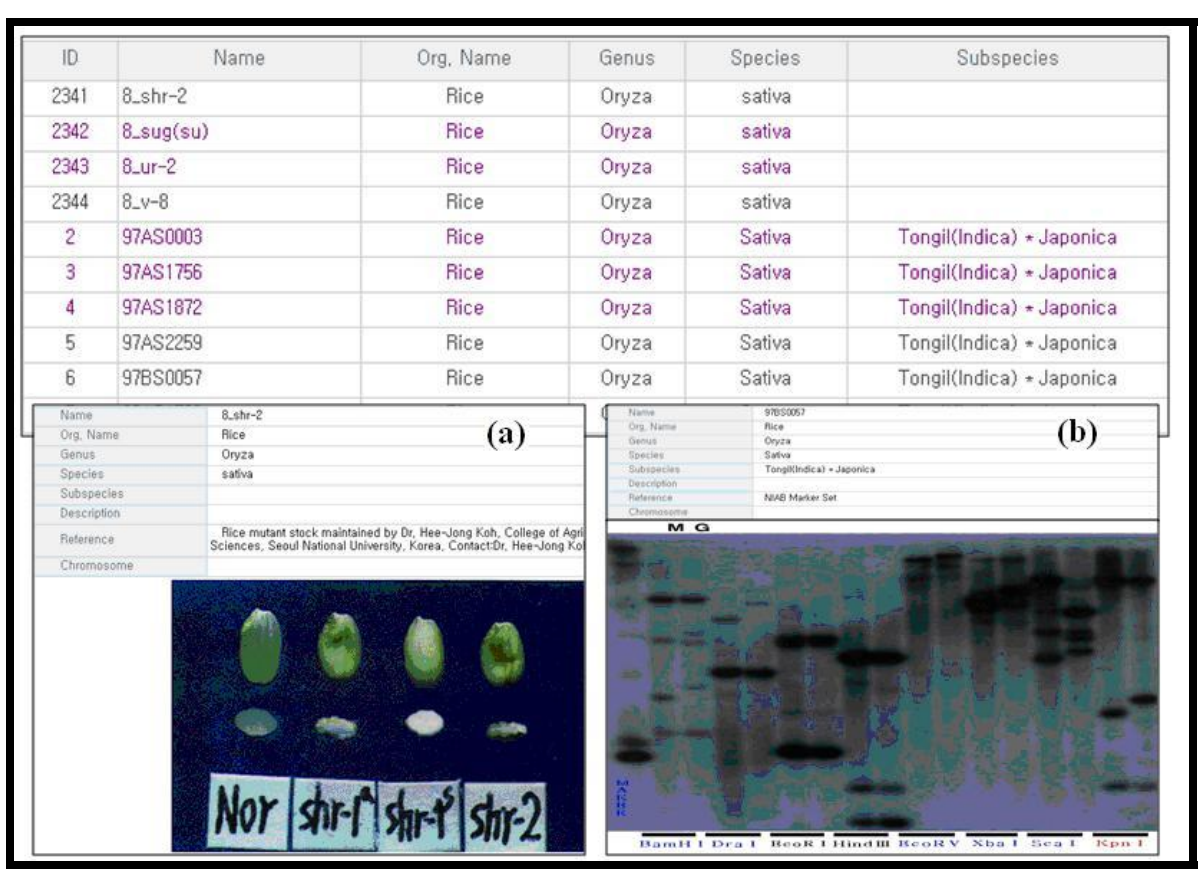

Figure 1: A snap shot of the database is given. The individual view table shows samples of searching result; (a) marker trait information table (ex: 8_shr-2, seed of rice) by clicking a specific marker name in the upper area (b) marker trait view table (ex: 97AS1872, RFLP-PCR image of Chinese cabbage).

\section{References:}

[01] http://www.gramene.org/markers/

[02] http://www.brassica-rapa.org /BGP/

[03] http://www.gramene.org/qtl/

[04] S. Katsumi et al., Nucleic Acids Res., 28: 97 (2000) [PMID: 10592192]

[05] Z. Huazong et al., Nucleic Acids Res., 35: 879 (2007) [PMID: 17142239]

[06] X. Qing et al., Bioinformatics, 24: 1011 (2008) [PMID: 18204061]
[07] R. A. Teutenico et al., Theor. Appl. Genet. 89: 885 (1994)

[08] J. S. Kim et al., Genetics, 174: 29 (2006) [PMID: 16988107]

[09] http://www.niab.go.kr/nabic/

[10] ftp://ftp.gramene.org/pub/gramene/release16/data/

[11] D. Kosambi, Ann. Eugen., 12: 172 (1944)

[12] J. B. S. Haldane, J Genet., 8: 299 (1919)

Citation: Tong et al., Bioinform b. Kangueane

License statement: This is an open-access article, which permits unrestricted use, distribution, and reproduction in any medium, for non-commercial purposes, provided the original author and source are credited. 